\title{
Médiévales
}

Langues, Textes, Histoire

49 | automne 2005

La paroisse, genèse d'une forme territoriale

\section{Territoires paroissiaux et construction de l'espace vernaculaire}

Parish territories and the construction of vernacular space

\section{Élisabeth Zadora-Rio}

\section{OpenEdition \\ 12 Journals}

\section{Édition électronique}

URL : https://journals.openedition.org/medievales/1306

DOI : 10.4000/medievales.1306

ISSN : $1777-5892$

Éditeur

Presses universitaires de Vincennes

Édition imprimée

Date de publication : 1 décembre 2005

Pagination : 105-120

ISBN : 2-84292-177-1

ISSN : 0751-2708

Référence électronique

Élisabeth Zadora-Rio, "Territoires paroissiaux et construction de l'espace vernaculaire », Médiévales [En ligne], 49 | automne 2005, mis en ligne le 05 mars 2008, consulté le 23 avril 2022. URL : http:// journals.openedition.org/medievales/1306; DOI : https://doi.org/10.4000/medievales.1306

Ce document a été généré automatiquement le 23 avril 2022.

Tous droits réservés 


\title{
Territoires paroissiaux et construction de l'espace vernaculaire
}

\author{
Parish territories and the construction of vernacular space
}

\author{
Élisabeth Zadora-Rio
}

1 Le maillage paroissial, qui a abouti, au prix d'un certain nombre de modifications, à la création des communes actuelles, est un découpage continu et homogène de l'espace, dont l'unité administrative de base est à l'échelle de la localité, celle de l'espace vernaculaire dans lequel s'inscrivent les pratiques religieuses et sociales des populations locales.

2 Contrairement à ce qui a été longtemps admis, la formation des territoires paroissiaux ne remonte pas aux premiers temps de la christianisation, mais résulte d'une longue élaboration. Ainsi que l'a montré M. Lauwers ${ }^{1}$, le processus de territorialisation de la paroisse, qui commence vers le $\mathrm{IX}^{\mathrm{e}}$ siècle, ne trouve son expression achevée dans le droit canon que vers 1250, dans la Somme dorée de Henri de Suse, dite Hostiensis. La paroisse y est définie comme un territoire circonscrit par des limites reconnues, qui constitue à la fois le cadre de vie des fidèles et le ressort dans lequel s'exerce le droit spirituel de l'église ${ }^{2}$.

La nouveauté radicale du maillage paroissial

3 La nouveauté radicale du réseau paroissial tient avant tout à son échelle : il constitue le premier maillage administratif à l'échelle de la localité.

4 La maîtrise spatiale de l'État romain, telle qu'elle se manifeste dans l'art de l'arpentage et le génie civil, à travers la mise en place des centuriations, du réseau routier, des aménagements hydrauliques, est si éclatante qu'on a eu tendance à penser qu'elle se manifestait également dans ses découpages administratifs. En réalité, les circonscriptions administratives de l'État romain, contrairement à celles des États contemporains, n'étaient pas contiguës et emboîtées, mais formaient un maillage lâche dont les éléments se recouvraient partiellement. Dans L'Inventaire du Monde, Cl. Nicolet 
a montré que jusqu'à la fin de la République, et même pendant une partie du règne d'Auguste,

«le cadre théorique de la vie civique et administrative n'est ni la Ville elle-même, dans son extension topographique, ni le village, le territoire ou la cité "municipale" mais une unité, un groupe qui n'est pas uniquement territorial, censitaire ou juridique, mais qui obéit à tous ces critères à la fois : la tribu. [...] L'empire, l'hégémonie de Rome constituent encore, jusqu'à la fin de la République, une série d'institutions complexes et enchevêtrées où ne règne aucun principe de base et dans lesquelles la territorialité n'est qu'une composante, mal coordonnée avec les autres. Car là encore préexiste et prédomine avant tout la nature du lien juridique qui unit telle communauté ou tel individu au peuple romain [...] il n'y a pas à cette époque, dans le droit public romain, de répartition territoriale des compétences des magistrats ${ }^{3}$ ".

C'est seulement sous le règne d'Auguste que le terme de "province » a pris, en Italie, une signification territoriale plus précise ${ }^{4}$. Les chevauchements et les enclaves étaient fréquents. Le territoire d'une cité pouvait s'étendre de part et d'autre d'une limite provinciale, comme dans le cas de Digne, à laquelle étaient rattachés des peuples des Alpes Maritimes ${ }^{5}$, et la continuité territoriale n'était pas non plus une règle, comme l'atteste l'exemple de Marseille qui avait des dépendances à Nice et à Vence, ou celui de Capoue qui possédait des terres en Crète ${ }^{6}$. Ainsi que le souligne P. Leveau, les contours des territoires n'ont cessé de changer au gré des transformations des statuts juridiques, et, à l'époque romaine, celles-ci n'ont pas pour objectif d'imposer un modèle unique de gestion de l'espace ${ }^{7}$. Les pagi étaient, en principe, des circonscriptions délimitées, à fonction fiscale et censitaire, qui faisaient l'objet de lustrations par les magistri pagi, mais leurs limites étaient souvent imprécises et leur répartition n'était ni régulière ni continue. Certains d'entre eux se trouvaient à cheval sur plusieurs cités et il arrivait également qu'un pagus empiète sur un autre ${ }^{8}$. Même si les vici, dans l'Antiquité, paraissent avoir bénéficié, au moins dans certains cas, d'une certaine autonomie administrative, leur territoire semble avoir été limité à l'agglomération et à ses environs immédiats ${ }^{9}$ et ils ne constituaient pas un découpage homogène et continu des circonscriptions administratives de rang supérieur, pagus ou cité.

6 Un resserrement du maillage administratif semble apparaître à l'époque carolingienne ${ }^{10}$, lorsque s'impose la référence spatiale à trois degrés - villa illa in pago illo, in condita illa - qui est attestée dans les Formules de Tours au milieu du viII ${ }^{11}$ et qui reste courante jusqu'à la fin du $\mathrm{x}^{\mathrm{e}}$ siècle, à ceci près que la condita est remplacée par la vicaria à partir des années 840. Contrairement à l'opinion généralement admise, le pagus, la vicaria et la villa, à l'époque carolingienne, ne constituaient pas des territoires emboîtés. Certaines vicariae s'étendaient sur deux pagi, tandis que des villae pouvaient appartenir à deux vigueries, voire à deux pagi : ainsi par exemple la villa de Han est-elle située en partie dans le pagus de Tours et la viguerie de Chinon, et en partie dans le pagus de Poitiers et la viguerie de Loudun ${ }^{12}$. Sans doute faut-il voir les vigueries comme des réseaux de dépendances plutôt que comme des territoires d'un seul tenant. En Touraine, au $\mathrm{x}^{\mathrm{e}}$ siècle, les vigueries dépendantes du comte de Blois et du comte d'Anjou constituaient deux réseaux d'autorité qui s'interpénétraient et se superposaient sur un même territoire ${ }^{13}$.

7 L'effacement du sens spatial de vicaria, qui est manifeste presque partout à partir de la fin $d u x^{e}$ ou du début du $x^{e}$ siècle, accompagne une recomposition territoriale à une échelle plus locale. Il est concomitant du phénomène de polarisation de l'espace autour 
des églises et des châteaux, qui a été bien mis en évidence par l'historiographie depuis une trentaine d'années, avec de nombreuses variantes régionales et sous des noms divers (incastellamento, encellulement, ensagrerament, inecclesiamento....). Il est bien établi désormais que c'est dans ce contexte qu'ont été mis en place les territoires paroissiaux. Mais quel a été le mode de structuration de l'espace, quel a été le processus de fixation des limites, et quelle prégnance, quel poids symbolique faut-il accorder à ces dernières? Cette question est importante dans la mesure où c'est sur ce postulat de l'intangibilité des limites paroissiales que se fonde l'utilisation de la méthode régressive pour reconstituer les territoires de villae carolingiennes, voire d'unités territoriales antérieures. L'archéologie du paysage, qui a révélé la très grande ancienneté de certains tracés de parcellaires, a accordé une place importante aux limites paroissiales, et elle a contribué à accréditer l'idée qu'elles pouvaient avoir été fixées à une époque très reculée. Certains archéologues, comme Ch. Taylor ou D. Bonney, ont tenté de montrer que les limites de paroisse avaient pu fossiliser des unités territoriales dont la constitution remontait à l'âge du fer, ou même à l'âge du bronze. D. Bonney, en particulier, a cherché à mettre en évidence une corrélation entre la répartition des sépultures sous tumulus et les limites de paroisses pour montrer que celles-ci avaient fossilisé des unités territoriales bien antérieures à la christianisation ${ }^{14}$. Dans la même perspective, A. Goodier a tenté d'établir un lien entre les lieux d'inhumation de la période anglo-saxonne et les limites des communes actuelles ${ }^{15}$. Si ces hypothèses ont été critiquées depuis, l'idée que les limites paroissiales ont fossilisé le tracé des domaines du haut Moyen Âge ou de l'Antiquité reste largement admise, notamment en Angleterre.

Le mode de structuration de l'espace vernaculaire

Deux modes de structuration spatiale, a priori très différents, doivent être envisagés.

9 Dans le premier cas, qui est celui des paroisses qui résultent d'un acte de fondation, la délimitation du territoire est contemporaine de la consécration de l'église. Ces espaces bornés, régis par la logique englobante de l'inclusion, sont conçus comme des contenants : la limite compte autant que le pôle central constitué par l'église ${ }^{16}$.

Dans le second cas, le territoire résulte d'une logique radiale d'extension du pôle ecclésial. Les limites territoriales sont définies progressivement, à la fois par les pratiques sociales et cultuelles des populations locales et par la rencontre ou le conflit avec d'autres pôles en expansion. La séparation entre le dedans et le dehors n'existe pas d'emblée, mais résulte d'un processus étalé dans le temps.

11 Le premier cas est connu par les actes de consécration qui ont donné lieu à un écrit. Leur répartition dans le temps et l'espace est très variable. La Catalogne en a conservé un corpus particulièrement important et précoce, puisque les premiers documents, dont des originaux, datent du $\mathrm{IX}^{\mathrm{e}}$ siècle ${ }^{17}$. Ces actes comportent généralement trois temps, présentés dans un ordre variable: 1) la dotation de l'église par les habitants, sous la forme d'une énumération de parcelles précisément localisées et délimitées ; 2) la consécration de l'église par l'évêque; 3) la délimitation de la paroisse: l'évêque définit un terminium à l'intérieur duquel les habitants devront verser les dîmes et les prémices à la nouvelle église. Ainsi par exemple à l'occasion de la consécration d'un groupe ecclésial à La Quar en 899 :

«Moi, l'évêque Nantigisus, je consacre ces églises et je leur confère le droit paroissial (parrohechiam) depuis le fleuve Azeste jusqu'au Mont Saint et depuis Les Quadrons jusqu'à la montagne de Petreio et jusqu'à la villa Mesata et au villare 
Lodovese avec ses limites et ses dépendances, afin que tous ceux qui travaillent sur ce territoire (terminium) apportent à ces églises toutes les dîmes et les prémices (qu'ils doivent) sur toutes choses ${ }^{18}$.»

12 Les limites décrites dans ces documents sont hétérogènes : elles sont marquées tantôt par des éléments linéaires (cours d'eau, routes), tantôt par le relief, ou par des repères ponctuels, tantôt enfin par le territoire d'une villa ou d'une paroisse voisine ${ }^{19}$. L'accent est mis sur la constitution d'une enveloppe territoriale dont les habitants seront affectés à la nouvelle église, plus que sur une délimitation précise déterminant l'appartenance des terres.

13 Le corpus catalan n'a guère d'équivalent ailleurs. À la même époque, on trouve dans d'autres régions des documents qui énumèrent les biens ou les dîmes constituant la dotation des églises, comme dans les cas bien connus de Milly en Mâconnais en 864-872, de Favars en Limousin en 897 ou de Cigogné en Touraine en $943^{20}$. Ces donations, formées de biens dispersés, étaient destinées à constituer le patrimoine de l'église mais pas un territoire paroissial. Elles n'impliquent pas de lien sacramentel et cultuel entre les contribuables et l'église à laquelle sont affectées leurs dîmes.

14 Un second ensemble d'actes de consécrations d'églises et de délimitations de paroisses, beaucoup plus diffus que le corpus catalan, apparaît aux XI ${ }^{\mathrm{e}}-\mathrm{XII}^{\mathrm{e}}$ siècles. Il s'inscrit dans la redéfinition des pouvoirs laïques et ecclésiastiques résultant du mouvement grégorien et il traduit l'importance nouvelle du rite de la consécration des autels et des cimetières, sous l'influence de la papauté, et notamment de Léon IX et Urbain II ${ }^{21}$. Les territoires paroissiaux attribués à l'église nouvellement consacrée ne sont pas toujours définis de façon exclusivement spatiale: ainsi les quelques paroisses fondées par l'évêque d'Angers dans le second tiers du XII ${ }^{\mathrm{e}}$ siècle, à la demande de petits seigneurs laïques, sont-elles caractérisées principalement par les liens de dépendance seigneuriale ${ }^{22}$. Même lorsque les territoires paroissiaux sont circonscrits, l'accent est mis sur les habitants qui sont attribués à la nouvelle église, plus que sur les terres. Ainsi, par exemple, lors de la fondation de la paroisse de La Roe, en 1098: avant de procéder à la consécration de l'autel et à la bénédiction du cimetière, à la prière de Robert d'Arbrissel, l'évêque d'Angers, Geoffroy de Mayenne, demanda au seigneur du lieu quels seraient les paroissiens de ce nouveau cimetière. Le seigneur désigna alors comme paroissiens ceux qui habitaient la forêt entre la voie de Grolet et le ruisseau de l'Usure ${ }^{23}$. Ces délimitations de paroisses faites à l'occasion de la consécration de l'église ont le caractère d'un manifeste, et sont porteuses d'un discours ecclésiologique. Elles dessinent indubitablement une enveloppe territoriale autour de l'église, mais elles n'impliquent pas un tracé linéaire et continu sur le terrain. Pas plus que dans les exemples catalans, elles n'induisent une sacralité et une intangibilité des limites paroissiales.

Dans le second cas de figure, le plus répandu, la constitution du territoire paroissial ne résulte pas d'un acte fondateur, contemporain de la consécration de l'église, mais de processus hétérogènes, qui font intervenir à la fois les contraintes topologiques qui pesaient sur la répartition des églises et les ressorts juridiques définis par les droits paroissiaux.

16 La répartition des églises paroissiales était soumise à deux contraintes, qui sont évoquées de manière récurrente depuis le $\mathrm{IX}^{\mathrm{e}}$ siècle jusqu'aux Temps modernes ${ }^{24}$, et qui agissaient en sens contraire. La première est la distance: la nécessité de se rendre fréquemment à l'église pour y assister aux offices, y recevoir les sacrements et inhumer 
les morts impliquait qu'aucun lieu habité ne soit trop éloigné pour que les femmes enceintes et les malades puissent faire le trajet ${ }^{25}$. L'analyse du maillage paroissial de Touraine à l'aide d'un Système d'Information Géographique (SIG) a montré que la distance entre les chefs-lieux était le facteur qui rendait le mieux compte de la superficie des territoires ${ }^{26}$. L'inscription dans l'espace des pratiques religieuses et sociales a donc joué un rôle déterminant dans leur constitution.

La seconde contrainte était celle de la viabilité du centre paroissial, définie principalement par la nécessité de pourvoir à l'entretien du bâtiment ecclésial et de son desservant. La dot de l'église, comme le bénéfice du prêtre, pouvaient être constitués de terres, de droits d'usages, ou d'une part des droits paroissiaux (droits de sépulture, oblations, offrandes à l'occasion de la bénédiction des mariages, des baptêmes, des confessions, des purifications, des offices dominicaux...). Selon la nature des biens qui composaient la dot de l'église ou le bénéfice du prêtre, il fallait donc que les terres soient assez vastes, ou la population suffisamment nombreuse.

Si la première de ces contraintes, déterminée par les pratiques sociales et cultuelles, tendait à multiplier les églises paroissiales et donc à réduire leur territoire, la seconde, d'ordre économique, exigeait au contraire que chaque paroisse ait une superficie et/ou une population suffisamment importante.

Les obstacles à la délimitation des territoires paroissiaux

À partir du deuxième tiers $\mathrm{du}_{\mathrm{XI}} \mathrm{e}^{\mathrm{e}}$ siècle, l'église devint un pôle de cristallisation de ressorts juridiques et fiscaux : c'est à l'église que se rattache une multitude de droits très divers, dont l'énumération exhaustive est impossible car leur liste n'est jamais close. Parmi ces droits, seule une partie fonde la paroisse, mais la distinction entre droits ecclésiastiques et droits seigneuriaux ne paraît pas toujours avoir été claire, même à la fin $\mathrm{du} \mathrm{XI} \mathrm{I}^{\mathrm{e}}$ et au XII ${ }^{\mathrm{e}}$ siècle, en dépit du mouvement grégorien. Les donations sont faites au titre de l'église (dono quicquid habeam in ecclesiam...), et les droits ecclésiastiques sont souvent énumérés en premier, mais ils sont traités sur le même plan que les droits seigneuriaux laïques.

20 Les territoires superposés que dessinent les décimaires, l'aire d'assistance aux offices, le bassin d'attraction du cimetière, sont tous centrés sur l'église, mais rien n'indique qu'ils soient précisément délimités ni qu'ils soient co-extensifs. Les recouvrements étaient nombreux.

- Le continuum juridique de l'église paroissiale à la chapelle

22 Les textes normatifs, depuis les conciles mérovingiens jusqu'aux Temps modernes, insistent sur l'opposition entre église paroissiale, d'une part, et chapelle ou oratoire, d'autre part. En fait, dans la pratique, les lieux de culte occupant une position intermédiaire et exerçant seulement une partie des droits paroissiaux étaient certainement très nombreux, et cette situation entraînait une superposition des ressorts juridiques contraire à toute délimitation tranchée des territoires.

23 Il est frappant de constater qu'aucun droit paroissial n'est absolument décisif : ni le presbiteratum (certaines chapelles avaient un desservant attitré avec le bénéfice attenant à la charge), ni les dîmes, qui pouvaient être attribuées à des lieux de culte de statuts divers, ni le baptême, car certaines églises avaient le droit de baptiser sans avoir celui d'inhumer, ni même le droit de sépulture, puisque des chapelles pouvaient être dotées d'un cimetière dûment consacré par l'évêque. 

l'Eglise métropolitaine de Tours fait état des sommes versées à la cathédrale par les paroisses du diocèse, et certaines d'entre elles sont toujours associées par deux, par trois ou par quatre ${ }^{30}$. Le lien entre ces associations fiscales et les territoires alternatifs des Temps modernes parait bien établi puisqu'on retrouve la plupart d'entre elles parmi les « virants et tournants » du XVIII siècle. Ces paiements groupés entre paroisses voisines révèlent sans doute une imbrication telle de leurs territoires que leurs redevances ne pouvaient être démêlées. Les territoires alternatifs correspondent à la notion de confins telle qu'elle a été définie par J. Lévy : confins se présentent comme une surface co-appartenante aux deux espaces en 
interface. L'analyse de deux espaces séparés par des confins ne peut se faire selon une approche classiquement topologique définissant le basculement brutal du dedans vers le dehors. Les confins constituent des espaces à forte composante topographique, c'està-dire marqués par la continuité et la présence de gradients là où l'idée de limite suggère plutôt, dans le sens commun, l'idée de rupture [...] Cela conduit à préciser la notion de confins: l'opposition ligne/surface ne suffit pas à la distinguer de la frontière, qui peut éventuellement s'exprimer par une bande, sous la forme d'un glacis. C'est seulement dans le cas d'une interpénétration entre les deux espaces concernés, c'est-à-dire d'une interface dont la distance qui sépare les deux éléments est négative, que la notion de confins prend tous son sens ${ }^{31}$. »

La cospatialité des territoires paroissiaux

33 Ces considérations paraissent en contradiction avec l'insistance sur les limites qu'on trouve dans le droit canonique à partir du milieu du XIII ${ }^{e}$ siècle ainsi que dans certains actes de la pratique ${ }^{32}$. Elles se heurtent surtout aux descriptions minutieuses que contiennent certains documents médiévaux qui sont de véritables procès-verbaux de délimitation. On constate cependant que ces derniers résultent presque toujours d'un conflit qui a obligé les parties à composer et à s'accorder sur un tracé de limites. Seul le tronçon contesté est ainsi rigidifié, mais son existence n'implique nullement que l'ensemble du territoire paroissial soit aussi précisément délimité : il faut certainement admettre la coexistence de zones de recouvrements territoriaux et de fragments de limites précisément tracées.

Dans le vocabulaire de la géographie, le terme de "cospatialité " ${ }^{33}$ caractérise l'articulation des couches spatiales "superposées " sur une même étendue. Selon la définition de J. Lévy, c'est, avec l'interface et l'emboîtement, l'une des familles de relations entre espaces, une des interspatialités. Dans chacune d'elles, "le lien est assuré par un type de connexion et de franchissement spécifique: la limite pour l'interface, le commutateur pour la cospatialité, qui constituent à leur tour des espaces. Pour l'emboîtement, le principe d'articulation entre un espace englobant et un espace englobé correspond assez bien au "saut d'échelle", notion classique en géographie " ${ }^{34}$. Dans cette perspective, l'église paroissiale, qui polarise de multiples territoires superposés, peut être considérée comme un commutateur de cospatialité.

Les tracés sont parfois restés flous jusqu'à la Révolution: lors de la création des communes en 1790, il a fallu parfois de longues enquêtes nécessitant le recours au témoignage des anciens pour fixer les bornes. Les arguments consignés dans les procèsverbaux sont les mêmes que ceux qu'on trouve dans les actes des $\mathrm{XI}^{\mathrm{e}}$-XII ${ }^{\mathrm{e}}$ siècles : ce qui détermine l'appartenance du territoire contesté à un chef-lieu plutôt qu'à un autre, c'est le fait que ses habitants y assistaient aux offices, y recevaient les sacrements, s'y faisaient inhumer, et y étaient inscrits au rôle d'imposition ${ }^{35}$.

Les territoires paroissiaux apparaissent comme des espaces en négociation permanente, constamment redéfinis par les usages. Ils sont le lieu d'une revendication d'appartenance de la part des populations locales, mais la définition de la communauté paroissiale dans son enveloppe territoriale l'emporte sur celle du tracé des limites. Les processions des Rogations, qui jouaient un rôle dans la mémorisation des limites, dessinaient un circuit autour de la paroisse, conditionné par les voies de circulation, et jalonné par des repères dont on inculquait le souvenir aux enfants. Il y a cependant un écart considérable entre ces circuits et un relevé cartographique, ainsi que le montrent les procès-verbaux de constitution des communes de 1790 qui reflètent le désarroi des 
populations locales confrontées à la nécessité de cartographier les limites territoriales ${ }^{36}$.

Dans l'ouvrage qu'il a consacré aux Frontières de France, D. Nordman observe l'apparition d'une nouvelle forme de territorialité, surtout à partir des années 1740-1760, et suggère que l'expérience des confins de la nation a pu servir de modèle pour le remodelage des circonscriptions internes :

« La gestion méthodique des confins ouvre le champ à une pensée unifiée qui tend à extraire définitivement le territoire de l'espace. Les droits hétérogènes et superposés, les liaisons complexes et contradictoires entre les lieux laissent la place à des unités physiquement homogènes, juxtaposées, qui recomposent sans interstices, sans chevauchement, l'ensemble des confins et du royaume tout entier ${ }^{37}$. »

L'introduction de la cartographie moderne ne s'est pas limitée à l'enregistrement des réalités géographiques. Elle a eu un rôle dynamique, ainsi que l'ont montré les travaux des géographes qui ont reconsidéré les rapports que la carte entretient avec le territoire qu'elle représente: «la cartographie s'inscrit dans les processus de territorialisation, au sens d'ensembles d'opérations techniques et symboliques par lesquels les sociétés marquent leurs espaces, se les approprient, en leur donnant sens et identité ${ }^{38}$. "

Il faut donc admettre que ce n'est pas avant la fin du XVIII ${ }^{\mathrm{e}}$ siècle et le développement de la cartographie moderne que les relations entre les territoires paroissiaux sont passées de la cospatialité à l'interface ${ }^{39}$. Si l'enveloppe territoriale des paroisses a sans doute été définie précocement dans sa masse, il n'en va pas de même des limites, dont la fixation a résulté d'un long processus et dont les fluctuations ont été nombreuses. Elles n'ont jamais eu le caractère intangible qu'on leur a prêté, à partir d'indices archéologiques attestant l'ancienneté de telle ou telle partie de leur tracé, et indûment extrapolés au territoire entier.

\section{NOTES}

1. Cf. l'article de M. Lauwers qui ouvre le présent volume.

2. "Quid sit parochia. Locus in quo degit populus alicui ecclesiae deputatus, certis finibus limitatus, et accepitur hic parochia quatenus spirituale ius ecclesiae se extendit, et in una determinatione plures baptismales esse non possunt » (Hostiensis, Summa aurea, lib. III (De parochiis)), Lyon 1537, XVI q 1, cité par M. Lauwers).

3. C. Nicolet, L'Inventaire du Monde, Paris, 1988, p. 202-204.

4. Ibid., p. 205.

5. Étude d'A. Chastagnol citée par P. Leveau, «Territorium urbis. Le territoire de la cité romaine et ses divisions : du vocabulaire aux réalités administratives ", Revue des Études Anciennes, 1993, p. 459-471.

6. P. Leveau, «Territorium urbis », op. cit., p. 468.

7. P. Leveau, « Les territoires. Un bilan des méthodes d'étude », dans Territoires celtiques. Espaces ethniques et territoires des agglomérations protohistoriques d'Europe centrale, 
Actes du xxiv ${ }^{e}$ colloque international de l'AFEAF (Martigues, 1-4 juin 2000), Paris 2002, p. 9-15 (p. 15).

8. M. Tarpin, Vici et pagi dans l'Occident romain, Rome, 2002, p. 188, 202-220.

9. P. Leveau, « Agglomérations secondaires et territoires en Gaule narbonnaise », Revue Archéologique de Narbonnaise, 26, 1993, p. 277-299 (p. 296-299), M. Tarpin, Vici et pagi, op. cit., p. 271-273.

10. Pour M. Lauwers et L. Rippart, le passage de l'Antiquité au Moyen Âge serait caractérisé par un processus de " déterritorialisation »: « Représentations et gestion de l'espace dans l'Occident médiéval (vexiiie siècles) », dans Rome et l'État moderne européen : une comparaison typologique, J.-P. Genet dir., École française de Rome (à paraître). Il me semblerait préférable de parler d'une recomposition territoriale.

11. Formulae Turonenses, dans Formulae merowingici et karolini aevi, MGH Legum $5 / 1, n^{\circ \mathrm{s}} 1$, $4,7,37$.

12. " est autem ipsa hereditas sita in villa qui dicitur Han, partim in pago Turonico et in vicaria Cainonensi, partimque in pago Pictavo et in vicaria Laudunensi » : ms. Paris, BnF, n.a.l. 1930, $\mathrm{n}^{\circ} 29, \mathrm{f}^{\circ}$ XVII vo-XIX $\mathrm{r}^{\circ}$ (début du xi $\mathrm{i}^{\mathrm{e}}$ siècle).

13. É. Zadora-Rio, «Territoires et limites", dans Des paroisses de Touraine aux communes d'Indre-et-Loire : formation et transformations des territoires, É. Zadora-Rio dir. (à paraître). 14. D. BONNEY, "Early boundaries and estates in southern England », dans P.H. SAWYER dir., Medieval Settlement. Continuity and change, Londres, 1976, p. 72-82.

15. A. GooDIER, « The formation of boundaries in Anglo-Saxon England: a statistical study ", Medieval Archaeology, XXVIII, 1984, p. 1-21.

16. D. Nordman, Frontières de France. De l'espace au territoire, XVI $-\mathrm{XIX}^{e}$ siècles, Paris, 1998 (Bibliothèque des Histoires), p. 514.

17. C. Barraut, « Les actes de consegracions d'esglesias del bispat d'Urgell (siglos IXXII) », Urgellia, 1, 1978, $n^{\circ}$ 11-182. D. Iogna-Prat, « Les moines et la "blanche robe d'églises" à l'âge roman ", dans Ante el Milenario del reinado de Sancho el Mayor. Un rey navarro par Espana y Europa, Actas de la XXX $\mathrm{XX}^{\mathrm{a}}$ Semana de Estudios Medievales (Estella, 14-18 julio 2003), Pampelune, 2004, p. 319-347. Je remercie D. Iogna-Prat d'avoir attiré mon attention sur ces documents.

18. « ego Nantigisus episcopus consecro ipsas ecclesias et trado eis parrohechiam de flumine Azeste usque Monte Sancto et de illos Quadrons usque in serra de Petreio et usque ad villa Mesata, et ipso villare Lodovese cum finibus et aiacenciis suis, ut omnes qui infra hunc terminium laboraverint cum omnes decimas et primicias de omni re ad iamdictas ecclesias semper occurrant $", i b i d ., \mathrm{n}^{\circ} 9$.

19. Ibid., $\mathrm{n}^{\circ} 11$ en $899, \mathrm{n}^{\circ} 13$ en $900, \mathrm{n}^{\circ} 19$ en $905, \mathrm{n}^{\circ} 27$ en $922, \mathrm{n}^{\circ} 30$ en 943.

20. G. Fournier, « La mise en place du cadre paroissial et l'évolution du peuplement », dans Cristianizzazione ed organizzazione ecclesiastica delle campagne nell'alto medioevo, Settimane di studio del Centro italiano di studi sull'alto medioevo, Spolète, 38, 1982 p. 495-575 ; F. Bange, « L'ager et la villa : structures du paysage et du peuplement dans la région mâconnaise à la fin du haut Moyen Âge (ix $-\mathrm{x}^{\mathrm{e}}$ siècles) », Annales ESC, 1984/3, p. 529-569 ; M. Aubrun, La Paroisse en France des origines au Xve siècle, Paris 1986, p. 199-200 ; É. Lorans, Le Lochois du haut Moyen Âge au XIII siècle, Tours, 1996, p. 73-75. 21. D. Iogna-Prat, « Léon IX, pape consécrateur », dans B.-M. Tock dir., Léon IX (à paraître) ; É. Zadora-Rio, « Lieux d'inhumation et espaces consacrés. Le voyage d'Urbain II en France (1095-1096) », dans A. Vauchez dir., Lieux sacrés, lieux de culte, sanctuaires, École française de Rome, 2000 (Collection de l'École française de Rome, 273), p. 197-213. 
22. É. Zadora-Rio, « Construction de châteaux et fondation de paroisses en Anjou aux $\mathrm{xi}^{\mathrm{e}}$-xii ${ }^{\mathrm{e}}$ siècles ", Archéologie Médiévale, IX, 1979, p. 115-125.

23. Cartulaire de La Roe, M. Hamon-Jugnet éd., Thèse de l'École nationale des chartes, 1971, nº 2 ; É. Zadora-Rio, « Construction de châteaux », art. cit., n. 17.

24. Exemples dans G. Fournier, « La mise en place du cadre paroissial », art. cit. ;

B. Maillard, « Les créations de paroisses rurales au xviii ${ }^{\mathrm{e}}$ siècle $: l^{\prime}$ exemple des diocèses de Tours et d'Angers ", dans Société et religion en France et aux Pays-Bas, $X V^{e}-X V I I I^{e}$ siècles. Mélanges en l'honneur d'Alain Lottier, Arras, 2000, p. 337-345.

25. M. Lauwers, « Circuit, cimetière, paroisse. Notes sur l'ancrage ecclésial des sites d'habitat (vii ${ }^{\mathrm{e}}$-Xiii ${ }^{\mathrm{e}}$ siècles) », dans Autour du "village » entre Seine et Rhin (IV ${ }^{e}$-XIII ${ }^{e}$ siècles), Actes du colloque de Louvain-la-Neuve, 16-17 mai 2003 (à paraître).

26. P. Chareille, X. Rodier, É. Zadora-Rio, « Analyse des transformations du maillage paroissial et communal en Touraine à l'aide d'un SIG », Histoire et Mesures, XIX/34, 2004, p. 317-343.

27. É. ZADORA-RIO, « The Making of Churchyards and Parish Territories in the Early Medieval Landscape of France and England in the 7th-12th Centuries : A Reconsideration », Medieval Archaeology, 47, 2003, p. 1-19.

28. J.-M. Gorry, Paroisses et communes de France. Indre-et-Loire, Paris, 1985, p. 14.

29. D. Pichot, Le Village éclaté. Habitat et société dans les campagnes de l'Ouest au Moyen Âge, Rennes, 2002, p. 235.

30. A. Longnon, Pouillés de la Province de Tours, Paris, 1903, p. 5-9. Je remercie B. Chevalier d'avoir attiré mon attention sur cette particularité du compte de 1290. 31. J. Lévy, "Confins », dans Dictionnaire de la géographie et de l'espace des sociétés, J. Lévy, M. Lussault dir., Paris, 2003, p. 197-198.

32. Par exemple en 1119-1125, à propos de l'église de Neuville, « ayant des limites propres et des bornes certaines, ainsi que l'exigent la loi et la coutume des autres paroisses » (« habens fines suos et terminos certos, sicut tenet lex et usus ceterarum parrochiarum ») : Chartes de Saint-Julien de Tours, L.J. Denis éd., Archives Historiques du Maine XII, Le Mans, 1913, $\mathrm{n}^{\circ} 68$.

33. J'ai emprunté l'utilisation de la notion de cospatialité à la stimulante communication de B. Cursente, R. Viader, « Les espaces du voisinage : entre données textuelles et paradigmes ethnologiques », présentée au colloque Espace et Société au Moyen Âge, organisé à Rosas (Espagne), en hommage à Monique Bourin (septembre 2004).

34. J. Lévy, "Cospatialité », dans Dictionnaire de la géographie, op. cit., p. 213-214 ; Id., «Interspatialité », ibid., p. 523.

35. J.-M. Gorry, « La reconstitution des limites communales de 1790 : méthodes et sources », dans Des paroisses de Touraine, op. cit., n. 11.

36. Ibid.

37. D. Nordman, Frontières de France, op. cit., p. 520-522.

38. F. Pousin, Figures de la ville et construction des savoirs, Paris, 2005 p. 53.

39. "Comme l'emboîtement et la cospatialité, l'interface est une famille de relations entre espaces, d'interspatialités. C'est sans doute la plus simple car elle se réalise horizontalement et "de face", sous forme d'un contact par juxtaposition. C'est aussi la plus communément identifiée, au point que beaucoup d'auteurs limitent les interactions entre espaces aux interfaces ", J. Lévy, dans Dictionnaire de la géographie, op. cit., p. 522. 


\section{RÉSUMÉS}

La nouveauté radicale du réseau paroissial, par rapport aux découpages administratifs de l'Antiquité et du haut Moyen Âge, tient à son échelle : c'est le premier maillage administratif à l'échelle de l'espace vernaculaire dans lequel s'inscrivent les pratiques religieuses et sociales des populations locales. Si la volonté d'enraciner la communauté paroissiale dans un territoire cohérent et précisément circonscrit est bien attestée aux $\mathrm{XII}^{\mathrm{e}}$-XIII ${ }^{\mathrm{e}}$ siècles, et si ses origines peuvent être tracées jusqu'au IX ${ }^{\mathrm{e}}$ siècle, sa mise en œuvre s'est heurtée à de nombreux obstacles, dus en particulier aux ressorts multiples des droits paroissiaux, qui n'étaient ni co-extensifs, ni précisément délimités. Les superpositions de territoires étaient nombreuses et ont pris fin seulement avec la cartographie moderne, lors de la création des communes en 1790.

Parish territories and the construction of vernacular space. The radical novelty of the parish network, as opposed to the administrative units of Antiquity and the early Middle Ages, lies in its scale : the parish network was the first administrative pattern on the scale of peasant communities, integrating the religious and social practices of local populations. If the will to anchor the parish community within a coherent and precisely delimited territory is clearly documented by the 12th-13th c., its enactment was fraught with obstacles, due in particular to the fact that the various forms of parochial jurisdiction were neither coterminous nor precisely defined geographically. Overlaps of territories were frequent and came to an end only with the birth of modern mapping and the delimitation of modern civil parishes in 1790.

\section{INDEX}

Mots-clés : paroisse, territoire, limite, cospatialité

Keywords : parish, territory, boundary

\section{AUTEUR}

ÉLISABETH ZADORA-RIO

CNRS, UMR 6173 Citères, Laboratoire Archéologie et Territoires, CNRS-Université de Tours, MSH Villes et Territoires, BP 60449, 37204 Tours cedex 03 